PROCEEDINGS OF THE

AMERICAN MATHEMATICAL SOCIETY

Volume 133, Number 10, Pages 3037-3046

S 0002-9939(05)08149-9

Article electronically published on April 20, 2005

\title{
FIXED POINTS OF NONEXPANSIVE MAPPINGS IN SPACES OF CONTINUOUS FUNCTIONS
}

\author{
T. DOMÍNGUEZ BENAVIDES AND MARÍA A. JAPÓN PINEDA
}

(Communicated by Jonathan M. Borwein)

\begin{abstract}
Let $K$ be a compact metrizable space and let $C(K)$ be the Banach space of all real continuous functions defined on $K$ with the maximum norm. It is known that $C(K)$ fails to have the weak fixed point property for nonexpansive mappings (w-FPP) when $K$ contains a perfect set. However the space $C\left(\omega^{n}+1\right)$, where $n \in \mathbb{N}$ and $\omega$ is the first infinite ordinal number, enjoys the w-FPP, and so $C(K)$ also satisfies this property if $K^{(\omega)}=\emptyset$. It is unknown if $C(K)$ has the w-FPP when $K$ is a scattered set such that $K^{(\omega)} \neq \emptyset$. In this paper we prove that certain subspaces of $C(K)$, with $K^{(\omega)} \neq \emptyset$, satisfy the w-FPP. To prove this result we introduce the notion of $\omega$-almost weak orthogonality and we prove that an $\omega$-almost weakly orthogonal closed subspace of $C(K)$ enjoys the w-FPP. We show an example of an $\omega$-almost weakly orthogonal subspace of $C\left(\omega^{\omega}+1\right)$ which is not contained in $C\left(\omega^{n}+1\right)$ for any $n \in \mathbb{N}$.
\end{abstract}

\section{INTRODUCTION}

Let $K$ be a compact metrizable space and let $C(K)$ be the Banach space of all real continuous functions defined on $K$ with the maximum norm. It is well known (see [14], [16]) that many topological properties of $K$ are strongly related to geometrical properties of $C(K)$. In this paper we are especially concerned with a geometrical property: The weak fixed point property for nonexpansive mappings. A Banach space $X$ is said to have the weak fixed point property for nonexpansive mappings (w-FPP) if every nonexpansive mapping $T$ defined from a nonempty convex weakly compact subset $M$ of $X$ into itself has a fixed point.

Whether or not every Banach space has the w-FPP was an open question for some years. In 1981, Alspach [1] solved this problem by proving that the Lebesgue space $L^{1}([0,1])$ fails to have the w-FPP. Despite the fact that no explicit example is known in any other Banach space, Alspach's example provides the failure of the w-FPP for any space containing isometrically $L^{1}([0,1])$. In particular, $C([0,1])$, which is universal for separable Banach spaces, fails to have the w-FPP. In fact, it is known [14. Main Theorem] that $C(K)$ isometrically contains $C([0,1])$ if and only if $K$ is a compact set which is not scattered (that is, $K$ contains a perfect non-void subset). Thus, $C(K)$ fails to have the w-FPP if $K$ is not scattered. On

Received by the editors May 30, 2004.

2000 Mathematics Subject Classification. Primary 47H09, 47H10, 46B20, 46B42, 46E05.

This research was partially supported by the DGES (research project BMF2000-0344-C02-C01) and the Junta de Andalucia (project 127).

(C)2005 American Mathematical Society Reverts to public domain 28 years from publication 
the other hand, it is known [5] that the space $C\left(\omega^{n}+1\right)$, where $n \in \mathbb{N}$ and $\omega$ is the first infinite ordinal number (for ordinal numbers we follow the notation in [16]), enjoys the w-FPP. Therefore, $C(K)$ also satisfies this property if $K^{(\omega)}=\emptyset$ where $K^{(\alpha)}$ denotes the $\alpha$-derived set of $K, \alpha$ being any ordinal number. It is unknown if $C(K)$ has the w-FPP when $K$ is a scattered set such that $K^{(\omega)} \neq \emptyset$. In Section 2, we will prove that a class of subspaces of $C(K)$ does satisfy the w-FPP. To do that, we introduce the notion of $\omega$-almost weak orthogonality and we prove that any $\omega$ almost weakly orthogonal closed subspace of $C(K)$ enjoys the w-FPP. This notion is a wide extension of the concept of weakly orthogonal Banach lattice, defined by Borwein and Sims 3. We will prove that the class of metrizable compact sets $K$ such that $C(K)$ is weakly orthogonal, is very strict. Actually, this class only contains those compact sets with finitely many accumulations points. However, we will show an example of an $\omega$-almost weakly orthogonal subspace $X$ of $C\left(\omega^{\omega}+1\right)$ which is not contained in any space $C\left(\omega^{n}+1\right)$ for $n \in \mathbb{N}$, i.e. for any topological compact space $K$, such that $X$ can be lattice isomorphically embedded in $C(K)$, we have $K^{(\omega)} \neq \emptyset$. Furthermore, we prove that $C\left(\omega^{n}+1\right)$ is $\omega$-almost weakly orthogonal, which means that our result is a strict extension of Corollary 3 in [5].

\section{Preliminaries}

In this section we introduce some known results related to the weak fixed point property, which will be used throughout this paper. For more details the reader may consult, for instance [7, [11. We also recall some classical topological and metric results concerning spaces of continuous functions.

Let $X$ be a Banach space and let $M$ be a nonempty convex weakly compact subset of $X$. Let $T: M \rightarrow M$ be a nonexpansive map (i.e. $\|T x-T y\| \leq\|x-y\|$ for every $x, y \in M)$ which is fixed point free. Using Zorn's Lemma we can find a subset $C$ of $M$ which is convex, weakly compact, $\operatorname{diam}(C)>0, T(C) \subset C$ and minimal in the following sense: There is no nonempty convex weakly compact proper subset of $C$ which is invariant under $T$.

On the other hand, it is well known that we can obtain a sequence $\left(x_{n}\right)$ of approximated fixed points for $T$, that is, $\lim _{n}\left\|x_{n}-T x_{n}\right\|=0$.

Goebel-Karlovitz' Lemma ([6, [10]). Let $C$ be a convex weakly compact subset of a Banach space $X$ and let $T: C \rightarrow C$ be a nonexpansive mapping. Assume that $C$ is minimal for $T$ and let $\left(x_{n}\right)$ be an approximated fixed point sequence. Then

$$
\lim _{n}\left\|x_{n}-x\right\|=\operatorname{diam}(C)
$$

for every $x \in C$.

We denote by $\ell_{\infty}(X)$ (respectively $c_{0}(X)$ ) the linear space of all bounded sequences (respectively all sequences convergent to zero) in the Banach space $X$ and we denote by $[X]$ the quotient space $\ell_{\infty}(X) / c_{0}(X)$. It is easy to check that the canonical quotient norm is defined by $\left\|\left[z_{n}\right]\right\|=\lim \sup _{n}\left\|z_{n}\right\|$, where $\left[z_{n}\right]$ is the equivalent class of $\left(z_{n}\right) \in \ell_{\infty}(X)$. By identifying $x \in X$ with the class $[(x, x, \ldots)]$ we can consider $X$ as a subset of $[X]$. If $C$ is a subset of $X$ we can define the set $[C]=\left\{\left[z_{n}\right] \in[X]: z_{n} \in C\right.$ for every $\left.n \in \mathbb{N}\right\}$. If $T$ is a mapping from $C$ into $C$, then $[T]:[C] \rightarrow[C]$ given by $[T]\left(\left[x_{n}\right]\right)=\left[T x_{n}\right]$ is a well-defined mapping. Note that if $\left(x_{n}\right)$ is an approximated fixed point sequence of $T$, its equivalent class $\left[x_{n}\right]$ is a fixed point for $[T]$. 
Lin's Lemma ([13]). Let $X$ be a Banach space and let $C$ be a weakly compact convex subset of $X$. Let $T: C \rightarrow C$ be a nonexpansive mapping and suppose that $C$ is a minimal $T$-invariant set. If $[W]$ is a nonempty closed convex subset of $[C]$ which is invariant under $[T]$, then

$$
\sup \left\{\left\|\left[w_{n}\right]-[x]\right\|:\left[w_{n}\right] \in[W]\right\}=\operatorname{diam}(C)
$$

for every $x \in C$.

Let $X$ be a Banach lattice. The Riesz angle for $X$ is defined by

$$
\alpha(X)=\sup \{\||x| \vee|y|\|:\|x\| \leq 1,\|y\| \leq 1\} .
$$

Borwein-Sims' Theorem (3]). Let $X$ be a weakly orthogonal Banach lattice with $\alpha(X)<2$. Then $X$ has the $w-F P P$.

In [3] it is proved that the space of all the real sequences $c$ is weakly orthogonal and therefore $c$ has the w-FPP. Borwein and Sims' result could suggest that many spaces of continuous functions $C(K)$ have the w-FPP. However, we will show that the class of compact sets $K$ where Borwein-Sims' Theorem can be applied is very strict.

We recall some well-known topological results.

Definition 2. Let $M$ be a topological space and let $A$ be a subset of $M$. The set $A$ is said to be perfect if it is closed and has no isolated points, i.e. $A$ is equal to the set of its own accumulation points. The space $M$ is said to be scattered if it contains no perfect nonvoid subset.

Cantor-Bendixson Theorem (16, page 148]). Let $A$ be a topological space. Then there exists an ordinal number $\alpha$ such that $A^{(\alpha+1)}=A^{(\alpha)}$. Moreover $A^{(\alpha)}=\emptyset$ if and only if $A$ is scattered.

Mazurkiewicz-Sierpiński Theorem ([16, page 155]). Every compact scattered first-countable space is homeomorphic to a countable compact ordinal.

In fact, the Mazurkiewicz-Sierpiński Theorem proves that $K$ is homeomorphic to $\omega^{\alpha-1} m+1$ if $\alpha$ is the smallest ordinal such that $K^{(\alpha)}=\emptyset$ and $m$ is the (finite) number of elements in $K^{(\alpha-1)}$. As a consequence $C(K)$ is isometric and order isomorphic to $C\left(\omega^{\alpha-1} m+1\right)$.

The following extension theorem will be a very important tool in this paper:

Borsuk-Dugundji Theorem ([16 page 365]). Let L be a closed nonempty subset of a metric space $K$. Then there exists a linear extension $\Lambda: C(L) \rightarrow C(K)$ such that $\|\Lambda\|=1$.

Remark. The mapping $\Lambda$ in the Borsuk-Dugundji Theorem does not necessarily preserve the order structure. However, if $f_{1}, \ldots, f_{n}$ are a finite family of functions in $C(L)$ and $x$ lies in $L$, we have

$$
\left|f_{1}(x)\right| \wedge \ldots \wedge\left|f_{n}(x)\right|=\left|\Lambda f_{1}(x)\right| \wedge \ldots \wedge\left|\Lambda f_{n}(x)\right| \leq\left\|\left|\Lambda f_{1}\right| \wedge \ldots \wedge\left|\Lambda f_{n}\right|\right\| .
$$

Taking supremum as $x$ runs in $L$ we obtain

$$
\left\|| f _ { 1 } | \wedge \ldots \wedge | f _ { n } | \left|\leq\left\||| \Lambda f_{1}|\wedge \ldots \wedge| \Lambda f_{n} \mid\right\| .\right.\right.
$$




\section{FiXed POINT RESUlts}

Definition 2.1. Let $X$ be a subspace of a Banach lattice. We say that $X$ is $w$ weakly orthogonal if for every weakly null sequence $\left(x_{n}\right) \subset X$ there exists some $p \in \mathbb{N}$ such that

$$
\liminf _{n_{p} \rightarrow \infty} \ldots \liminf _{n_{1} \rightarrow \infty}\left\|\left|x_{n_{p}}\right| \wedge \ldots \wedge\left|x_{n_{1}}\right|\right\|=0 .
$$

We say that $X$ is $w$-almost weakly orthogonal if for every weakly null sequence $\left(x_{n}\right) \subset B_{X}$, where $B_{X}$ is the closed unit ball in $X$, there exists some $p \in \mathbb{N}$ such that

$$
\liminf _{n_{p} \rightarrow \infty} \ldots \liminf _{n_{1} \rightarrow \infty} \|\left|x_{n_{p}}\right| \wedge \ldots \wedge\left|x_{n_{1}}\right|||<1 / p .
$$

Theorem 2.3. Let $X$ be a w-almost weakly orthogonal closed subspace of $C(K)$ where $K$ is a metrizable compact space. Then $X$ has the $w$-FPP.

Proof. By contradiction we assume that $X$ fails to have the w-FPP. Thus we can find a convex weakly compact set $C$ of $X$ with $\operatorname{diam}(C)=1$ and such that $C$ is minimal invariant for a nonexpansive mapping $T$. Let $\left(x_{n}\right)$ be an approximated fixed point sequence that, by translation, we can consider to be weakly null. Since $0 \in C$ and diam $(C)=1$ we know that $\left(x_{n}\right)$ is in $B_{X}$. Since $X$ is $\omega$-almost weakly orthogonal, there exists some $p \in \mathbb{N}$ (depending on $\left(x_{n}\right)$ ) and $c<1 / p$ such that

$$
\liminf _{n_{p} \rightarrow \infty} \ldots \liminf _{n_{1} \rightarrow \infty} \|\left|x_{n_{p}}\right| \wedge \ldots \wedge\left|x_{n_{1}}\right| \mid<c .
$$

Next, we are going to construct $\left(x_{n_{s}(1)}\right)_{s \in \mathbb{N}},\left(x_{n_{s}(2)}\right)_{s \in \mathbb{N}}, \ldots,\left(x_{n_{s}(p)}\right)_{s \in \mathbb{N}}$ subsequences of $\left(x_{n}\right)$ satisfying the following properties:

$$
\begin{gathered}
\lim _{s \rightarrow \infty}\left\|x_{n_{s}(i)}-x_{n_{s}(j)}\right\|=1 \quad \text { for every } i, j \in\{1, \ldots, p\}, i \neq j, \\
\lim _{s \rightarrow \infty}\left\|\left|x_{n_{s}(1)}\right| \wedge \ldots \wedge\left|x_{n_{s}(p)}\right|\right\| \leq c .
\end{gathered}
$$

Indeed, fix $s \in \mathbb{N}$. From (1) we can find $n_{s}(1) \in \mathbb{N}$ large enough such that

$$
\liminf _{n_{2} \rightarrow \infty} \ldots \liminf _{n_{p} \rightarrow \infty} \|\left|x_{n_{s}(1)}\right| \wedge\left|x_{n_{2}}\right| \wedge \ldots \wedge\left|x_{n_{p}}\right| \mid<c+\frac{1}{s} .
$$

From Goebel-Karlovitz' Lemma we know that $\lim _{n \rightarrow \infty}\left\|x_{n}-x_{n_{s}(1)}\right\|=1$, and thus we can find $n_{s}(2)$ such that

$$
\left\|x_{n_{s}(2)}-x_{n_{s}(1)}\right\| \geq 1-\frac{1}{s} .
$$

Given $k \in\{1, \ldots, p-1\}$ suppose that we have found $n_{s}(1), n_{s}(2), \ldots, n_{s}(k)$ positive integers such that

$$
\begin{gathered}
\| x_{n_{s}(i)}-x_{n_{s}(j)} \mid \geq 1-\frac{1}{s}, \quad i, j \in\{1, \ldots, k\}, i \neq j, \quad \text { and } \\
\liminf _{n_{k+1} \rightarrow \infty} \ldots \liminf _{n_{p} \rightarrow \infty} \|\left|x_{n_{s}(1)}\right| \wedge \ldots \wedge\left|x_{n_{s}(k)}\right| \wedge\left|x_{n_{k+1}}\right| \wedge \ldots \wedge\left|x_{n_{p}}\right| \mid<c+\frac{1}{s} .
\end{gathered}
$$

From Goebel-Karlovitz's Lemma we know that $\lim _{n \rightarrow \infty}\left\|x_{n}-x_{n_{s}(i)}\right\|=1$ for every $i \in\{1, \ldots, k\}$. Hence, we can find $n_{s}(k+1)$ such that $\left\|x_{n_{s}(k+1)}-x_{n_{s}(i)}\right\| \geq 1-\frac{1}{s}$ for every $i \in\{1, \ldots, k\}$ and

$$
\liminf _{n_{k+2} \rightarrow \infty} \ldots \liminf _{n_{p} \rightarrow \infty}||\left|x_{n_{s}(1)}\right| \wedge \ldots \wedge\left|x_{n_{s}(k+1)}\right| \wedge\left|x_{n_{k+2}}\right| \wedge \ldots \wedge\left|x_{n_{p}}\right| \mid<c+\frac{1}{s} .
$$


Thus, by induction, we can construct $n_{s}(1), n_{s}(2), \ldots, n_{s}(p)$ positive integers such that

$$
\left\|x_{n_{s}(i)}-x_{n_{s}(j)}\right\| \geq 1-\frac{1}{s}, \quad i, j \in\{1, \ldots, p\}, i \neq j
$$

and

$$
\|\left|x_{n_{s}(1)}\right| \wedge \ldots \wedge\left|x_{n_{s}(p)}\right|||<c+\frac{1}{s} .
$$

Inductively using the above argument for $s=1,2, \ldots$, we construct $\left(x_{n_{s}(1)}\right)_{s \in \mathbb{N}}$, $\left(x_{n_{s}(2)}\right)_{s \in \mathbb{N}}, \ldots,\left(x_{n_{s}(p)}\right)_{s \in \mathbb{N}}$ subsequences of $\left(x_{n}\right)$. It is clear that these subsequences are approximated fixed point sequences, and from (4) and (5) we deduce properties (2) and (3).

Now consider the space $[X]$ and define $[T]:[C] \rightarrow[C]$ as in Section 1 . We denote by $\left[x_{1}\right], \ldots,\left[x_{p}\right]$ the equivalence class of $\left(x_{n_{s}(1)}\right)_{s \in \mathbb{N}},\left(x_{n_{s}(2)}\right)_{s \in \mathbb{N}}, \ldots,\left(x_{n_{s}(p)}\right)_{s \in \mathbb{N}}$, respectively. Then $\left[x_{1}\right], \ldots,\left[x_{p}\right]$ are fixed points for $[T]$ and $\left\|\left[x_{i}\right]-\left[x_{j}\right]\right\|=1$ for every $i, j \in\{1, \ldots, p\}, i \neq j$. Define the following subset of $[X]$ :

$$
[W]:=\left\{\left[t_{n}\right] \in[C]:\left\|\left[t_{n}\right]-\left[x_{i}\right]\right\| \leq \frac{p-1}{p} \text { for every } i \in\{1, \ldots, p\}\right\} .
$$

The set $[W]$ is nonempty since $\frac{1}{p} \sum_{i=1}^{p}\left[x_{i}\right] \in[W]$. It is also clear that $[W]$ is convex, closed and $[T]$-invariant.

Fix $\left[w_{s}\right] \in[W]$. It is not difficult to check that for every $s \in \mathbb{N}$ we have

$$
\left|w_{s}\right| \leq\left|w_{s}-x_{n_{s}(1)}\right| \vee \ldots \vee\left|w_{s}-x_{n_{s}(p)}\right|+\left|x_{n_{s}(1)}\right| \wedge \ldots \wedge\left|x_{n_{s}(p)}\right|
$$

which implies, using the triangular inequality in $X$ and the definition of $M$-space, that

$$
\left\|w_{s}\right\| \leq\left\|w_{s}-x_{n_{s}(1)}\right\| \vee \ldots \vee\left\|w_{s}-x_{n_{s}(p)}\right\|+\left\|\left|x_{n_{s}(1)}\right| \wedge \ldots \wedge\left|x_{n_{s}(p)}\right|\right\| .
$$

Taking limit as $s$ goes to infinity we obtain

$$
\begin{gathered}
\left\|\left[w_{s}\right]\right\|=\limsup _{s}\left\|w_{s}\right\| \leq\left\|\left[w_{s}\right]-\left[x_{1}\right]\right\| \vee\left\|\left[w_{s}\right]-\left[x_{2}\right]\right\| \vee \ldots \vee\left\|\left[w_{s}\right]-\left[x_{p}\right]\right\| \\
\quad+\lim _{s \rightarrow \infty}\left\|x_{n_{s}}(1) \wedge \ldots \wedge x_{n_{s}}(p)\right\| \leq \frac{p-1}{p}+c<1 .
\end{gathered}
$$

Thus sup $\left\{\left\|\left[w_{s}\right]\right\|:\left[w_{s}\right] \in[W]\right\}<1$, which contradicts Lin's Lemma since $0 \in C$.

\section{Some $\omega$-Almost Weak orthogonal spaces}

We will look for properties assuring that a subspace of $C(K)$ is $\omega$-almost weakly orthogonal. We start giving a characterization of the spaces $C(K)$ which are $\omega$-weak orthogonal.

Theorem 3.1. Let $K$ be a compact metrizable space. Then, the following conditions are all equivalent:

(1) $C(K)$ is $\omega$-almost weakly orthogonal,

(2) $C(K)$ is $\omega$-weakly orthogonal,

(3) $K^{(\omega)}=\emptyset$.

Proof. We first prove that $(3) \Rightarrow(1)$. Assume that $K^{(\omega)}=\emptyset$ and $\left\{f_{n}\right\}$ is a weakly null sequence in $C(K)$. We claim that

$$
\liminf _{n_{p} \rightarrow \infty} \ldots \liminf _{n_{1} \rightarrow \infty}\left\|\left|f_{n_{p}}\right| \wedge \ldots \wedge\left|f_{n_{1}}\right|\right\|=0
$$


if $K^{(p)}=\emptyset$. If $K^{(\omega)}=\emptyset$ we know (by compactness) that there exists $p \in \mathbb{N}$ such that $K^{(p)}=\emptyset$ and the result follows. We use an induction argument on $p$. It is clear that the claim holds if $p=1$. Assume that the claim holds for $f_{n} \in C(L)$, where $L$ is any compact set such that $L^{(p-1)}=\emptyset$ and let $K$ be a compact set with $K^{(p)}=\emptyset$. Take $\left(f_{n}\right)$ a weakly null sequence in $C(K)$.

Since $K^{(p-1)}$ is a finite set, we can write $K^{(p-1)}=\left\{t_{1}, \ldots, t_{m}\right\}$. Fix a positive integer $n_{p}$ and choose open neighborhoods $V_{i}$ of $t_{i}, i=1, \ldots, m$, such that $\mid f_{n_{p}}(t)-$ $f_{n_{p}}\left(t_{i}\right) \mid<\frac{1}{n_{p}}$ if $t \in V_{i}$. Set $L=K \backslash \bigcup_{i=1}^{m} V_{i}$, which is a compact set with $L^{(p-1)} \subset$ $K^{(p-1)} \cap L=\emptyset$. Consider the weakly null sequence $\left(g_{n}\right) \subset C(L)$ defined by $g_{n}(t)=$ $f_{n}(t)$ for every $t \in L$. Therefore, according to the induction hypotheses we know that

$$
\liminf _{n_{p-1} \rightarrow \infty} \ldots \liminf _{n_{1} \rightarrow \infty} \|\left|g_{n_{p-1}}\right| \wedge \ldots \wedge\left|g_{n_{1}}\right|||=0 .
$$

Let $t \in K$. If $t \in L$ we have

$$
\left|f_{n_{p}}\right| \wedge\left|f_{n_{p-1}}\right| \wedge \ldots \wedge\left|f_{n_{1}}\right|(t) \leq\left|f_{n_{p-1}}\right| \wedge \ldots \wedge\left|f_{n_{1}}\right|(t) \leq\left\|\left|g_{n_{p-1}}\right| \wedge \ldots \wedge\left|g_{n_{1}}\right| \mid\right\|
$$

If $t \in K \backslash L=\bigcup_{i=1}^{m} V_{i}$ we also have

$$
\left|f_{n_{p}}\right| \wedge\left|f_{n_{p-1}}\right| \wedge \ldots \wedge\left|f_{n_{1}}\right|(t) \leq\left|f_{n_{p}}\right|(t) \leq \max _{i=1, \ldots, m}\left|f_{n_{p}}\left(t_{i}\right)\right|+\frac{1}{n_{p}} .
$$

Taking the supremum we have

$$
\left\|\left|f_{n_{p}}\right| \wedge \ldots \wedge\left|f_{n_{1}}\right|\right\| \leq \max \left\{\left\|\left|g_{n_{p-1}}\right| \wedge \ldots \wedge\left|g_{n_{1}}\right|\right\|, \max _{i=1, \ldots, m}\left|f_{n_{p}}\left(t_{i}\right)\right|+\frac{1}{n_{p}}\right\} .
$$

Finally, taking limits we obtain

$$
\liminf _{n_{p} \rightarrow \infty} \ldots \liminf _{n_{1} \rightarrow \infty}\left\|\left|f_{n_{p}}\right| \wedge \ldots \wedge\left|f_{n_{2}}\right|\right\| \leq \lim _{n_{p} \rightarrow \infty}\left(\max _{i=1, \ldots, m}\left|f_{n_{p}}\left(t_{i}\right)\right|+\frac{1}{n_{p}}\right)=0 .
$$

Next, we prove that $(2) \Rightarrow(3)$. Assume that $C(K)$ is an $\omega$-almost weakly orthogonal Banach lattice. Then $K$ is a scattered set. Indeed, otherwise we obtain a contradiction because $C(K)$ contains $L^{1}([0,1])$ and has the w-FPP according to Theorem 2.3. So, assume by contradiction that $K$ is scattered and $K^{(\omega)} \neq \emptyset$. In this case, we can assume that $\omega^{\omega}+1$ is a subset of $K$ and using the Borsuk-Dugundji Theorem (see the remark after this theorem), it suffices to prove that $C\left(\omega^{\omega}+1\right)$ is not $\omega$-almost weakly orthogonal. To do that, we can use the sequence $\left(f_{n}\right)$ constructed in [15] for the space $C(Q)$, where $Q$ is a compact subset of $\omega^{\omega}+1$. Indeed, this sequence is a weakly null $\{0,1\}$-valued sequence which satisfies that for any finite sets of integers $\left\{m_{1}<m_{2}<\ldots<m_{m_{1}+1}\right\}$ there exists $t \in Q$ such that

$$
f_{m_{1}}(t)=\ldots=f_{m_{m_{i}+1}}(t)=1 \text {. }
$$

Thus, for any $p \in \mathbb{N}$ we have

$$
\liminf _{n_{1} \rightarrow \infty} \ldots \liminf _{n_{p} \rightarrow \infty}\left\|\left|f_{n_{1}}\right| \wedge \ldots \wedge\left|f_{n_{p}}\right|\right\|=1
$$

which shows that $C(Q)$ is not $\omega$-almost weakly orthogonal. Again using the BorsukDugundji Theorem we obtain that $C(K)$ is not $\omega$-almost weakly orthogonal, a contradiction. Finally, since (1) obviously implies (2), we conclude the proof. 
Remarks. (1) The metrizability assumption of $K$ can be replaced in Theorem 3.1 by a much weaker notion. Indeed, if $K$ is compact, scattered, $K^{(\omega)} \neq \emptyset$ and each point $t \in K$ has a neighborhood basis consisting of a decreasing (possibly transfinite) sequence $\left\{U_{\alpha}\right\}_{\alpha<\tau}$ of sets, then there is a surjective map from $K$ onto a compact ordinal $\omega^{\alpha} n+1$ where $\alpha \geq \omega$ (see [12, page 32]). This fact implies that, again, we can assume $\omega^{\omega}+1$ is contained in $K$ and the proof of Theorem 3.1 equally holds. Therefore, Theorem 3.1 can be applied, for instance, when $K$ is any compact ordinal number bigger than the first uncountable ordinal $\omega_{1}$ to prove that $C(K)$ is not $\omega$-almost weakly orthogonal.

(2) It is easy to check the first part of the proof of Theorem 3.1 (for the special case $p=2$ ) proves that $C(K)$ is weakly orthogonal when $K^{(2)}=\emptyset$. We will prove after Theorem 3.3 that this is a characterization of weak orthogonality for spaces $C(K)$.

Using Theorem 2.3 and Theorem 3.1 we easily derive a result which is equivalent to Corollary 3 in [5].

Corollary $3.2([5])$. Let $K$ be a compact set with $K^{(w)}=\emptyset$. Then $C(K)$ has the $w-F P P$.

Remark. When $K$ is an infinite metric compact space, it is known (see 2]) that $K^{(w)}=\emptyset$ if and only if $C(K)$ is isomorphic to $c_{0}$. Thus, we can state the above corollary as follows: If $C(K)$ is isomorphic to $c_{0}$, then $C(K)$ has the $w$-FPP. This result is, in some sense, surprising, because an isomorphic property implies the existence of fixed points for a nonexpansive mapping which is, clearly, an isometric property. (Recall 4 that $L_{1}[0,1]$, which fails to have the w-FPP, can be renormed in such a way that the new space has normal structure (which implies the w-FPP), and this new norm is as close (in the Banach-Mazur distance) to the original norm as wanted.) Moreover, it was known [3] that any Banach space $X$ isomorphic to $c_{0}$ such that the Banach-Mazur distance between $X$ and $c_{0}$ is less than 2, has the w-FPP. However, Corollary 3.2 assures the w-FPP for a class of spaces which are isomorphic to $c_{0}$ where Banach-Mazur distance is arbitrarily large. Indeed, if $K^{(p)} \neq \emptyset$ and $K^{(p+1)}=\emptyset$, then the Banach-Mazur distance $d\left(c_{0}, C(K)\right)$ is greater than $p$ (see [2, Remark 1]).

Theorem 3.1 shows that Theorem 2.3 does not properly extend the class of the spaces $C(K)$ which enjoy the w-FPP. However, Theorem 2.3 is a strict improvement of the results in [5] for subspaces of $C(K)$. Indeed, the following theorem shows an example of an $\omega$-almost weakly orthogonal subspace of $C\left(\omega^{\omega}+1\right)$ which is not contained in any $C\left(\omega^{n}+1\right)$ for $n \in \mathbb{N}$. (This example also shows that the notions of $\omega$-almost weak orthogonality and $\omega$-weak orthogonality are different.)

Theorem 3.3. There exists a subspace of $C\left(\omega^{\omega}+1\right)$ which is $\omega$-almost weakly orthogonal, and it is not order isomorphically contained in any space $C\left(\omega^{n}+1\right)$ for $n \in \mathbb{N}$.

Proof. Denote $A_{p}=\left[\omega^{p-1}+1, \omega^{p}\right]=\left(\omega^{p-1}, \omega^{p}+1\right)$ which is a clopen subset of $\omega^{\omega}+1$. To simplify the notation, we shall write $\left\langle m_{1}, \ldots, m_{k}\right\rangle$ to denote the ordinal $\omega^{p-1} m_{1}+\ldots+\omega^{p-k} m_{k}$. Consider the subset $B_{p}$ of $A_{p}$ defined by

$B_{p}=\left\{\alpha=\left\langle m_{1}, \ldots, m_{k}\right\rangle: k=1,2, \ldots, p, 1<m_{1}<m_{2}<\ldots<m_{k-1}<m_{k}\right\} \cup\left\{\omega^{p}\right\}$.

We claim that $B_{p}$ is a closed subset of $A_{p}$. Indeed, assume that $t=\lim _{s \rightarrow \infty} t_{s}$, where $t_{s}=\left\langle m_{1}(s), \ldots, m_{k(s)}(s)\right\rangle \in B_{p}$. There is a subsequence, again denoted 
$t_{s}$, such that for any $i=1, \ldots, p$ we have that either $\lim _{s} m_{i}(s)=\infty$ or $m_{i}(s)$ is a constant, say $m_{i}$. If for every $i=1, \ldots, p$ we have the second alternative, the result is clear. Otherwise, assume that $j=\min \left\{i: \lim _{s} m_{i}(s)=\infty\right\}$. Thus, $t=\left\langle m_{1}, \ldots, m_{j-1}+1\right\rangle \in B_{p}$ if $j>1$, or $t=\omega^{p}$ if $j=1$. Hence $B_{p}$ is a closed subset of $A_{p}$ and so it is a compact metrizable space.

For any positive integer $n>1$, we define a sequence $\left\{h_{n}\right\}$ in $C\left(B_{p}\right)$ in the following way: $h_{n}\left(\left\langle m_{1}, \ldots, m_{k}\right\rangle\right)=1$ if $n \in\left\{m_{1}, \ldots, m_{k-1}, m_{k}-1\right\}$ and $h_{n}(t)=0$ otherwise. We claim that $h_{n}$ is a continuous function. It suffices to prove that $\left.B_{n, p}=\left\{\left\langle m_{1}, \ldots, m_{k}\right\rangle\right) \in B_{p}: n \in\left\{m_{1}, \ldots, m_{k-1}, m_{k}-1\right\}\right\}$ is an open and closed subset of $B_{p}$. To prove that $B_{n, p}$ is a closed subset of $B_{p}$, assume that $t_{s}=$ $\left\langle m_{1}(s), \ldots, m_{k(s)}(s)\right\rangle$ is a sequence in $B_{n, p}$ (i.e. $m_{1}(s)<m_{2}(s)<\ldots<m_{k(s)}(s)$ and $\left.n \in\left\{m_{1}(s), \ldots, m_{k(s)-1}, m_{k(s)}(s)-1\right\}\right)$ convergent to $t=\left\langle m_{1}, \ldots, m_{k}\right\rangle \in B_{p}$. Without loss of generality, we can assume that there exists $j>1$ such that $m_{i}(s)=$ $m_{i}$ for any $s, i=1, \ldots j-1$ and $m_{j}(s) \rightarrow_{s} \infty$. Thus $n<m_{i}(s)$ for any $i \geq j$ and $s$ large enough which implies that $n$ belongs to $\left\{m_{1}, \ldots, m_{j-1}\right\}$ and $t=\left\langle m_{1}, \ldots\right.$, $\left.m_{j-1}+1\right\rangle$ belongs to $B_{n, p}$. On the other hand, to prove that $B_{n, p}$ is an open subset of $B_{p}$, assume that $t_{s} \rightarrow_{s} t=\left\langle m_{1}, \ldots, m_{k}\right\rangle \in B_{n, p}$, where $t_{s} \in B_{p}$. For $s$ large enough we have $t_{s}=t$ or $t_{s}=\left\langle m_{1}, \ldots, m_{k-1}, m_{k}-1, m_{k+1}(s), \ldots\right\rangle \in B_{n, p}$.

It is easy to check that the sequence $\left\{h_{n}\right\}$ is weakly null. Furthermore for any $t \in B_{p}$ we have that card $\left\{n \in \mathbb{N}: h_{n}(t) \neq 0\right\} \leq p$ and for any choice of distinct positive integers $n_{1}, \ldots, n_{p}$ greater than 1 , there exists $t \in B_{p}$ such that $\|\left|h_{n_{1}}\right| \wedge \ldots \wedge\left|h_{n_{p}}\right| \mid=1$. Denote

$$
K=\bigcup_{p=1}^{\infty} B_{p} \cup\left\{\omega^{\omega}\right\} .
$$

We claim that $K$ is a closed subset of $\omega^{\omega}+1$ and so it is a metrizable compact space. Indeed, assume that $\left(t_{n}\right)$ is a sequence in $K$ convergent to $t \in \omega^{\omega}+1$. If there exists $k \in \mathbb{N}$ such that $t_{n} \in \bigcup_{p=1}^{k} B_{p}$ for every $n \in \mathbb{N}$, then $t \in K$ because $\bigcup_{p=1}^{k} B_{p}$ is a closed subset of $\omega^{\omega}+1$. Otherwise, for any $k \in \mathbb{N}$ there exists $n_{k}$ with $t_{n_{k}} \notin \bigcup_{p=1}^{k} B_{p}$ which implies that $t=\omega^{\omega}$. Define $h_{n}^{(p)}: K \rightarrow\{0,1\}$ by $h_{n}^{(p)}(t)=h_{n}(t)$ if $t \in B_{p}$ and $h_{n}^{(p)}(t)=0$ otherwise. Since $B_{p}$ is a clopen subset of $K$, we know that $h_{n}^{(p)}$ is a weakly null sequence in $C(K)$. We define

$$
f_{n}=h_{n}^{(1)}+\sum_{p=2}^{n} \frac{1}{4 p} h_{n}^{(p)}
$$

which is also a weakly null sequence in $C(K)$. Let $X$ be the closed space generated by $\left(f_{n}\right)$. Then, $X$ is a subspace of $C(K)$ which is not $\omega$-weakly orthogonal because for any $p \in \mathbb{N}$ we have

$$
\begin{aligned}
& \liminf _{n_{1}} \ldots \liminf _{n_{p}}\left\|\left|f_{n_{1}}\right| \wedge \ldots \wedge\left|f_{n_{p}}\right|\right\|_{C(K)} \\
\geq & \frac{1}{4 p} \liminf _{n_{1}} \ldots \liminf _{n_{p}}\left\|\left|h_{n_{1}}^{(p)}\right| \wedge \ldots \wedge\left|h_{n_{p}}^{(p)}\right|\right\|_{C(K)} \\
= & \frac{1}{4 p} \liminf _{n_{1}} \ldots \liminf _{n_{p}}\left\|\left|h_{n_{1}}\right| \wedge \ldots \wedge\left|h_{n_{p}}\right|\right\|_{C\left(B_{p}\right)}=\frac{1}{4 p} .
\end{aligned}
$$

Thus $X$ is not $\omega$-weakly orthogonal and by Theorem $3.1, X$ is not order isomorphic to any subspace of $C\left(\omega^{n}+1\right)$. However $X$ is $\omega$-almost weakly orthogonal. Indeed, 
let $f$ be a mapping in span $\left(f_{n}\right)$, i.e. $f=\lambda_{2} f_{2}+\ldots+\lambda_{n} f_{n}$. For $a \in B_{p}$ we have

$$
f(a)=\lambda_{n_{1}} f_{n_{1}}(a)+\ldots+\lambda_{n_{q}} f_{n_{q}}(a)
$$

for some $1<n_{1}<\ldots<n_{q}, q \leq p$ because card $\left(\left\{n \in \mathbb{N}: f_{n}(a) \neq 0\right\}\right) \leq p$. Thus

$$
|f(a)| \leq\left(\left|\lambda_{n_{1}}\right|+\ldots+\left|\lambda_{n_{q}}\right|\right) \frac{1}{4 p} \leq \frac{1}{4} \max \left\{\left|\lambda_{n_{i}}\right|: i=1, \ldots, q\right\} .
$$

Hence for some $i \in\{1, \ldots, q\}$ we have $\left|\lambda_{n_{i}}\right| \geq 4|f(a)|$. Since there exists $a_{1} \in B_{1}$ $\left(a_{1}=n_{i}+1\right)$ satisfying $f_{n_{i}}\left(a_{1}\right)=1$ and $f_{n_{j}}\left(a_{1}\right)=0$ if $j \neq i$, we have $|f(a)|=$ $\left|\lambda_{n_{i}}\right| \geq 4|f(a)|$. Thus

$$
\left\|f_{\mid B_{p}}\right\| \leq \frac{1}{4}\|f\| .
$$

Assume that $\left(g_{n}\right)$ is a weakly null sequence in $B_{X}$. By approximation, we can assume that $\left(g_{n}\right)$ is in span $\left(f_{n}\right)$. Since $C\left(B_{1}\right)$ is order isometrically contained in $C(\omega+1)$ and this space is weakly orthogonal, we know that

$$
\liminf _{n_{1} \rightarrow \infty} \liminf _{n_{2} \rightarrow \infty} \|\left|g_{n_{1} \mid B_{1}}\right| \wedge\left|g_{n_{2} \mid B_{1}}\right| \mid=0 .
$$

On the other hand

$$
\left\|g_{n_{i} \mid K \backslash B_{1}}\right\| \leq \frac{1}{4}, \quad i=1,2 .
$$

Thus

$$
\liminf _{n_{1} \rightarrow \infty} \liminf _{n_{2} \rightarrow \infty} \|\left|g_{n_{1}}\right| \wedge\left|g_{n_{2}}\right||| \leq \frac{1}{4}<\frac{1}{2}
$$

and $X$ is $\omega$-almost weakly orthogonal.

Since any compact metrizable set $K$ such that $K^{(2)} \neq \emptyset$ contains homeomorphically $\omega^{2}+1$ and so $B_{2}$, the proof of Theorem 3.3 and the Borsuk-Dugundji Theorem assures us that $C(K)$ is not weakly orthogonal if $K^{(2)} \neq \emptyset$. Thus, using the remark after Theorem 3.1 we can state the following result showing that the class of compact metrizable spaces $K$ such that $C(K)$ is weakly orthogonal is very strict.

Theorem 3.4. Let $K$ be a metrizable compact space. Then the following properties are equivalent:

(1) $C(K)$ is a weakly orthogonal Banach lattice.

(2) $K^{(2)}=\emptyset$.

Remark. In [8] the notion of convex orthogonality is defined and used to prove that the space $c$ has the w-FPP. We recall that a Banach space $E$ is said to be orthogonally convex if for every weakly null sequence $\left\{x_{n}\right\}$ in $E$ with $D\left(x_{n}\right)>0$ there exists a positive number $\lambda$ such that $A_{\lambda}\left(x_{n}\right)<D\left(x_{n}\right)$ where

$$
\begin{gathered}
D\left(x_{n}\right)=\limsup _{m \rightarrow \infty} \limsup _{n \rightarrow \infty}\left\|x_{n}-x_{m}\right\|, \\
A_{\lambda}\left(x_{n}\right)=\limsup _{m \rightarrow \infty} \limsup _{n \rightarrow \infty} \max \left\{\|z\|: z \in M_{\lambda}\left(x_{n}, x_{m}\right)\right\}, \\
M_{\lambda}(x, y)=\{z \in E: \max \{\|z-x\|,\|z-y\|\} \leq 1 / 2\|x-y\|(1+\lambda)\} .
\end{gathered}
$$

Again using the sequence constructed in $B_{2}$ in the proof of Theorem 3.3 it is not difficult to check that this notion for a space $C(K)$ is also equivalent to weak orthogonality. 


\section{ACKNOWLEDGEMENT}

The authors are very grateful to A. Pelczynski for his useful suggestions.

\section{REFERENCES}

1. D. E. ALSPACH, A fixed point free nonexpansive map, Proc. Amer. Math. Soc. 82 (1981), 423-424. MR0612733 (82j:47070)

2. C. BESSAGA and A. PELCZYNSKI, Spaces of continuous functions (IV) (On isomorphical classifications of spaces $C(S)$ ). Studia Math. 19 (1960), 53-62. MR0113132(22:3971)

3. J. M. BORWEIN and B. SIMS, Nonexpansive mappings on Banach lattices and related topics, Houston J. Math. 10 (1984), 339-356. MR.0763236 (86e:47059)

4. D. van DULTS, Equivalent norms and fixed point property for nonexpansive mappings J. London Math. Soc. 25 (1982), 139-144. MR.0645871 (83e:47040)

5. J. ELTON, P.K. LIN, E. ODELL, and S. SZAREK, Remarks on the fixed point problem for nonexpansive maps, Contemporary Math. 18 (1983), 87-120. MR0728595 (85d:47059)

6. K. GOEBEL, On the structure of the normal invariant sets for nonexpansive mappings, Annal. Univ. Mariae Curie-Sklodowska 29 (1975), 70-72. MR0461226 (57:1211)

7. K. GOEBEL and W. KIRK, Topics in Metric Fixed Point Theory. Cambridge University Press, Cambridge, 1990 MF1074005 (92c:47070)

8. A. JIMÉNEZ MELADO and E. LLORENS FUSTER, A sufficient condition for the fixed point property, Nonlinear Anal. 20(7) (1993), 849-853. MR.1214748 (94a:47097)

9. S. KAKUTANI, Concrete representation of abstract (M)-spaces. (A characterization of the space of continuous functions). Ann. Math. 4 (1941), 994-1024. MR0005778 (3:205g)

10. L.A. KARLOVITZ, On nonexpansive mappings, Proc. Amer. Math. Soc. 55 (1976), 321-325. MR0405182 (53:8976)

11. W. KIRK and B. SIMS (editors), Handbook in Metric Fixed Point Theory. Kluwer Academic Publishers 2001. MR.1904271 (2003b:47002)

12. H. E. LACEY, The Isometric Theory of Classical Banach Spaces. Springer-Verlag 1974. MR0493279 (58:12308)

13. P.K. LIN, Unconditional bases and fixed points of nonexpansive mappings. Pacific J. Math. 116 (1985), 69-76. MR0769823 (86c:47075)

14. A. PELCZYNSKI and Z. SEMADENI, Spaces of continuous functions (III). Studia Math. 18 (1959), 211-222. MR0107806 (21:6528)

15. A. PELCZYNSKI and W. SZLENK, An example of a non-shrinking basis. Rev. Roum. Math. Pures Appl. X (10) (1965), 961-966. MR0203432 (34:3284)

16. Z. SEMADENI, Banach Spaces of Continuous Functions. Polish Scientific Publishers, Warzawa, 1971. MR0296671 (45:5730)

Departamento de Análisis Matemático, University of Seville, P.O. Box 1160, 41080Seville, Spain

E-mail address: tomasd@us.es

Departamento de Análisis Matemático, University of Seville, P.O. Box 1160, 41080Seville, Spain

E-mail address: japon@us.es 